Mathematical Modelling and Analysis

Volume 22 Number 3, May 2017, 389-407

https://doi.org/10.3846/13926292.2017.1315344

(c) Vilnius Gediminas Technical University, 2017
Publisher: Taylor\&Francis and VGTU

http://www.tandfonline.com/TMMA

ISSN: $1392-6292$

eISSN: 1648-3510

\title{
Relaxation Oscillations in Singularly Perturbed Generalized Liénard Systems with Non-Generic Turning Points
}

\section{Huan Hu, Jianhe Shen, Zheyan Zhou and Zhonghui Ou}

\author{
School of Mathematics and Computer Science, Fujian Normal University \\ 350007 Fuzhou, China \\ E-mail: jhshen@f jnu.edu.cn
}

Received April 20, 2016; revised March 28, 2017; published online May 15, 2017

\begin{abstract}
Based on the asymptotic analysis technique developed by Eckhaus [Lecture Notes in Math., vol. 985, pp 449-494. Springer, Berlin, 1983], this paper aims to study the existence and the asymptotic behaviors of relaxation oscillations of regular and canard types in a singularly perturbed generalized Liónard system with a non-generic turning point. The singularly perturbed Liónard system considered in this paper is very general and numerous real world models like some biological ones can be rewritten in the form of this system after a series of transformations. Under certain conditions, we rigorously prove the existence of regular relaxation oscillations and canard relaxation oscillations under the specific parameter conditions. As an application, two biological models, namely, a FitzHugh-Nagumo model and a twodimensional predator-prey model with Holling-II response are studied, in which, the existence of regular relaxation oscillations and canard relaxation oscillations as well as the bifurcation curves are obtained.
\end{abstract}

Keywords: singular perturbation, relaxation oscillation, canard, non-generic turning point, generalized Liénard system.

AMS Subject Classification: 34C26; 34E17; 34E20.

\section{Introduction}

Liénard equation is a classical and important nonlinear oscillator, modeling nonlinear vibrations in the damped, one-degree-of-freedom systems. It can be viewed as a generalization of van der Pol equation, and has found numerous applications in various real world situations including electronic devices and biology, etc., see e.g. [3], [11], [13], [17] and [19]. For a long time, the existence, uniqueness, number and profile of limit cycles in Liénard equations are always the important and valuable topics in the field of ODEs.

Liénard equation reads,

$$
\frac{d^{2} x}{d t^{2}}+\mu f(x, b) \frac{d x}{d t}+g(x, a)=0,
$$


where $f$ and $g$ are sufficiently smooth functions, $a \in R^{n}$ and $b \in R^{m}$ are the bifurcation parameters, and $\mu \in R$ is related to the strength of damping. After the Liénard transformation, this equation becomes

$$
\left\{\begin{array}{l}
\frac{d x}{d t}=y-\mu F(x, b), \\
\frac{d y}{d t}=-g(x, a),
\end{array}\right.
$$

where $F(x, b)=\int_{0}^{x} f(s, b) d s$. If $g(x, a)$ is linear in $x$, this system is called classical Liénard system, and otherwise, it is called generalized one.

When $\mu$ is small, i.e. when the damping is small, in this case, Liénard system can be viewed as Hamiltonian-perturbation system. By using Abel integral etc., bifurcation of limit cycles has been studied extensively, especially when $f$ and $g$ are polynomials, see e.g. Han et al. [12] and Xiong et al. [23].

When $\mu$ is large, then after a new time scale $\tau=t / \mu$ is introduced, Liénard equation can be changed to

$$
\epsilon \frac{d^{2} x}{d \tau^{2}}+f(x, b) \frac{d x}{d \tau}+g(x, a)=0,
$$

which is a singular perturbation equation since the highest-order derivative has been multiplied by a small parameter, where $0<\epsilon=\frac{1}{\mu^{2}} \ll 1$. On the Liénard plane, this singularly perturbed equation is equivalent to a slow-fast dynamical system, namely,

$$
\left\{\begin{array}{l}
\epsilon \frac{d x}{d \tau}=y-F(x, b), \\
\frac{d y}{d \tau}=-g(x, a) .
\end{array}\right.
$$

Roussarie [20] pointed out that the singularly perturbed Liénard systems consists of the boundary of the so-called Liénard space.

The limit cycles occurring in singularly perturbed Liénard systems are relaxation oscillations, characterized by the presence of phases in the cycle with different time scales: A phase of slow change is followed by a short phase of rapid change where the system jumps to the next stage of slow variation. In this direction, non-standard analysis was first introduced to detect canard relaxation oscillations in van der Pol equation [1]. Then Eckhaus [10] pointed out that classical asymptotic analysis method is also capable of identifying canard relaxation oscillations and regular relaxation oscillations in singularly perturbed Liénard systems. Krupa and Szmolyan distinguished the generic jump and canard points for general singular perturbation system. Now it is well known that the results obtained from classical asymptotic analysis method are closely related to which from blow-up technique, see van Gils, Krupa and Szmolyan [22]. Based on geometric singular perturbation method and blow-up technique, Dumortier and Roussarie [8], [9] developed a tool, namely, the slow divergence integral. By this integral, the maximal number of limit cycles is related to its non-degenerate zeroes, and more limit cycles than expected by the conjecture of Lins et al. [18] in the classical singularly perturbed Liénard systems had been found in [4] and [7]. The cyclicity in the predator-prey models with Holling-III and IV responses had also been obtained, see Li and Zhu [17]. 
In all the above-mentioned works, it was assumed that the turning points on the critical curves are generic (non-degenerate). When the critical curve have non-generic (degenerate) turning points, the work on this topic is relatively rare. By following the method of Eckhaus [10], Li [15] detected canards with or without heads in a singularly perturbed Liénard system of the following form,

$$
\left\{\begin{array}{l}
\epsilon \frac{d x}{d t}=y-F(x), \\
\frac{d y}{d t}=a-x,
\end{array}\right.
$$

in which, it was assumed that $x=0$ is a $m$-order turning point on the critical curve $y=F(x)$, where $m$ is an even number. Then Li [16] extended this approach to a more general system, namely,

$$
\left\{\begin{array}{c}
\epsilon \frac{d x}{d t}=y-F(x) \\
\frac{d y}{d t}=-g(x, y, a)
\end{array}\right.
$$

where the parametric conditions for the existence of canards were obtained. By using also asymptotic analysis method, Chen et al. [2] further studied a more general singularly perturbed Liénard system, namely,

$$
\left\{\begin{array}{l}
\epsilon \frac{d x}{d t}=y-F(x)+\epsilon h(x, a, \epsilon), \\
\frac{d y}{d t}=-g(x, y, a, \epsilon),
\end{array}\right.
$$

where it was also assumed that $x=0$ is a degenerate turning point. Chen et al. [2] proved the existence of canard solutions, and optimized the canard curve based on a new asymptotic lemma.

In this paper, we will extend the asymptotic analysis method of Eckhaus [10] to study the birth of relaxation oscillations in the following singularly perturbed generalized Liénard system,

$$
\left\{\begin{array}{l}
\epsilon \frac{d x}{d t}=g(y)-f(x), \\
\frac{d y}{d t}=h(x, y, a),
\end{array}\right.
$$

in which, we assume that the critical curve admits a non-generic turning point, where $0<\epsilon \ll 1$ is the singular parameter, while $a \in R$ is a regular parameter (named also breaking parameter by following Dumoutier [5]), $g, f$ and $h$ are sufficiently smooth functions. We demonstrate the birth of relaxation oscillations of regular and canard types in (1.1) and give the corresponding parameter conditions.

Obviously, system (1.1) is more general than those studied in the previous papers. In fact, when $g(y)=y$, it reduces to the systems considered before. In literatures, system (1.1) is still called generalized Liénard system. Locally, system (1.1) can be changed to the simpler systems mentioned above after 
a series of transformations, but globally it is impossible. So if one aims to study system (1.1) globally, we must face to system (1.1). This is the first reason why we consider system (1.1) in this article. Secondly, a great deal of biological models can be changed to the form of system (1.1) after a series of transformations involving the dependent and independent variables as well as the parameters, see e.g. De Maesschalck [3], Hus and Shi [13] and Li and Zhu [17]. Thus, the study on the slow-fast dynamics such as the existence of relaxation oscillations in these models is reduced to study system (1.1), which is one of the main topics of the present paper.

The remaining parts of this paper are organized as follows. In section 2, several necessary assumptions and lemmas are given. In sections 3 and 4 , we respectively study the existence of regular relaxation oscillations and canard relaxation oscillations in system (1.1) and give the corresponding parametric curves. In section 5 , based on the conclusions obtained in the two previous sections, we consider two biological models, namely, a FitzHugh-Nagumo equation and a two-dimensional predator-prey model with Holling-II response, in which, we show the existence of regular relaxation oscillations and canard relaxation oscillations in these models and give the critical parameter values.

\section{Preliminaries and assumptions}

For system (1.1), the critical curve is defined by $g(y)=f(x)$, on which, the slow flows evolve according to the following equation

$$
g^{\prime}(y) h(x, y, a)=f^{\prime}(x) \frac{d x}{d t} .
$$

To fix the directions of the slow flows and the fast fibers, we need the following assumptions.

(A1) $x=x_{1}<0$ and $x=x_{2}=0$ are respectively the generic and nongeneric extreme points of the critical curve satisfying $f^{\prime}\left(x_{1}\right)=0, f^{\prime \prime}\left(x_{1}\right)<0$, $f^{\prime}(0)=\cdots=f^{(m-1)}(0)=0$, and $f^{(m)}(0)>0$, where $m>2$ is an even number.

(A2) $f^{\prime}(x)<0$ for $x \in\left(x_{1}, 0\right)$, and $f^{\prime}(x)>0$ for $x \in\left[C_{1}, x_{1}\right) \cup\left(0, C_{2}\right]$, where $C_{1}$ and $C_{2}$ are constants chosen such that $C_{1}<x_{1}<0<C_{2}, f\left(C_{1}\right)<0$ and $f\left(C_{2}\right)>f\left(x_{1}\right)$.

(A3) $h(x, y, a)=0$ has a unique root $x=\varphi(y, a)$ satisfying:

1) $h(x, y, a)<0$ when $x>\varphi(y, a)$, and $h(x, y, a)>0$ when $x<\varphi(y, a)$,

2) when $y \in\left(f(0), f\left(x_{1}\right)\right)$ and $a \in\left(a_{1}, a_{0}\right)$, we have $\varphi(y, a) \in\left(x_{1}, 0\right)$, when $a \rightarrow a_{0}$ and $y \rightarrow f(0)$, we get $\varphi(y, a) \rightarrow 0$, and when $a \rightarrow a_{1}$ and $y \rightarrow f\left(x_{1}\right)$, we obtain $\varphi(y, a) \rightarrow x_{1}$.

(A4) $g(0)=f(0)$ and, $g^{\prime}(y)>0$ for any $y \in R$.

(A5) $h\left(0,0, a_{0}\right)=0$ and $\frac{\partial h}{\partial a}\left(0,0, a_{0}\right) \neq 0$.

Remark 1. Under the assumption (A1), the critical curve is S-shape (cubiclike), where the right and left branches are attracting, while the middle one is repelling, see Figure 1. 
Remark 2. Under the assumptions (A1) to (A3), system (1.1) possesses an equilibrium point, namely, $\left(x_{e}, y_{e}\right)=\left(\varphi(y, a), g^{-1} f(\varphi(y, a))\right)$, where $g^{-1}$ denotes the inverse function of $g$. When $a \in\left(a_{1}, a_{0}\right)$, this equilibrium point lies on the middle branch of the critical curve. In this case, we mark the directions of the slow flows and the fast fibers in Figure 1a.

Remark 3. When $a \rightarrow a_{0}$ or $a \rightarrow a_{1}$, the equilibrium point of the system coincides with the extreme points of the critical curve, see Figure 1b, where the directions of the slow flows and the fast fibers when $a \rightarrow a_{0}$ are stated.

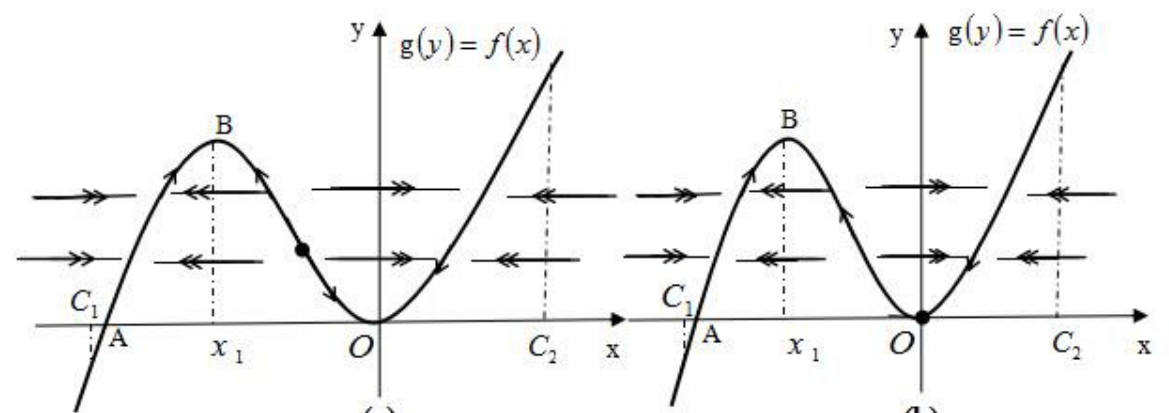

(a)

(b)

Figure 1. The directions of the slow flows (single arrows) and the fast fibers (double arrows). In the figure, the large dots denote the equilibriums.

(a): The case when $a \in\left(a_{1}, a_{0}\right)$; (b): The case when $a=a_{0}$.

In the following, two lemmas needed in the proof of our main results are given firstly. Consider

$$
\left\{\begin{array}{l}
\frac{d Y}{d \tau}=F(Y, \tau, \epsilon), \quad F: R^{n} \times R \times R \rightarrow R^{n}, \\
Y(0, \epsilon)=Y_{0},
\end{array}\right.
$$

in which, 1) $F$ is continuous and uniformly bound on

$$
G=\{Y \mid Y \in \bar{D}\} \times\{\tau \mid 0 \leq \tau<A\} \times\left\{\epsilon \mid 0<\epsilon \leq \epsilon_{0}\right\},
$$

where $D \subset R^{n}$ is a bounded region; 2) $F$ is Lipchitz-continuous with respect to $Y$ in $G$.

Lemma 1. (Eckhaus [10]) Let $Y^{(1)}$ and $Y^{(2)}$ be respectively the solutions of

$$
\begin{aligned}
& Y^{(1)}(\tau, \epsilon)=Y_{0}^{(1)}+\int_{0}^{\tau} F_{1}\left[Y^{(1)}\left(\tau^{\prime}, \epsilon\right), \tau^{\prime}, \epsilon\right] d \tau^{\prime}, \\
& Y^{(2)}(\tau, \epsilon)=Y_{0}^{(2)}+\int_{0}^{\tau} F_{2}\left[Y^{(2)}\left(\tau^{\prime}, \epsilon\right), \tau^{\prime}, \epsilon\right] d \tau^{\prime} .
\end{aligned}
$$

If the following conditions are satisfied: (i) $Y_{0}^{(1)} \in D_{0} \subset D, Y_{0}^{(2)} \in D_{0} \subset D$ and $\left|Y_{0}^{(1)}-Y_{0}^{(2)}\right| \leq \delta_{0}(\epsilon)$ with $\delta_{0}(\epsilon)=o(1)$, 
(ii) $\left|F_{1}-F_{2}\right| \leq \delta_{f}(\epsilon)$ when $(Y, \tau, \epsilon) \in G$, where $\delta_{f}(\epsilon)=o(1)$,

(iii) $Y^{(2)}(\tau, \epsilon)$ exists for $0 \leq \tau \leq T$, where $T<A$ and $Y^{(2)} \in D_{0}$, then $Y^{(1)}(\tau, \epsilon)$ exists for $0 \leq \tau \leq T$ and satisfies

$$
\left|Y^{(1)}(\tau, \epsilon)-Y^{(2)}(\tau, \epsilon)\right|=o\left(\delta_{0}(\epsilon)\right)+o\left(\delta_{f}(\epsilon)\right) .
$$

Lemma 2. (Eckhaus [10]) Let $Y^{(2)}(\tau, \epsilon)$ be a valid approximation to $Y^{(1)}(\tau, \epsilon)$, i.e.

$$
Y^{(1)}(\tau, \epsilon)-Y^{(2)}(\tau, \epsilon)=o(1), 0 \leq \tau \leq T,
$$

where $T$ is a constant which can be taken to be arbitrarily large, then there must be a $\tilde{\delta}(\epsilon)=o(1)$ such that the above approximation holds for $0 \leq \tau \leq \frac{1}{\tilde{\delta}(\epsilon)}$. Similarly, if $Y^{(2)}(\tau, \epsilon)$ is an approximation to $Y^{(1)}(\tau, \epsilon)$ for $0<d \leq \tau \leq T$, where $d$ can be chosen to be arbitrarily small, then there must be a $\hat{\delta}(\epsilon)=o(1)$ such that the approximation holds for $\hat{\delta}(\epsilon) \leq \tau \leq T$.

Lemma 3. (Chen et al. [2]) Let $m$ be an even number, $\varphi(x)$ and $\psi(x)$ are both defined on $[a, b]$. If

1) $\varphi(x) e^{n \psi(x)}$ is integrable on $[a, b]$ for any $n \geq n_{0}$,

2) $\psi(x)$ has a unique maximal point $x=\xi \in(a, b)$, and $\sup _{x \in[\alpha, \beta]} \psi(x)<\psi(\xi)$ holds on any closed subinterval $[\alpha, \beta] \subset[a, b], \xi \notin[\alpha, \beta]$,

3) near $x=\xi, \psi^{(m)}(x)$ is continuous and satisfies $\psi^{\prime}(\xi)=\psi^{\prime \prime}(\xi)=\cdots=$ $\psi^{m-1}(\xi)=0$, but $\psi^{m}(\xi)<0$,

4) $\varphi(x)$ is continuous at $x=\xi, \varphi(\xi) \neq 0$, then as $n \rightarrow+\infty$, we have

(i) $\int_{a}^{b} \varphi(x) e^{n \psi(x)} d x=\frac{2}{m} \varphi(\xi)\left(\frac{-m !}{n \psi^{(m)}(\xi)}\right)^{\frac{1}{m}} e^{n \psi(\xi)} \Gamma\left(\frac{1}{m}\right)\left(1+O\left(\frac{1}{n}\right)\right)$, where $\Gamma\left(\frac{1}{m}\right)=\int_{0}^{+\infty} e^{-x} x^{\frac{1}{m}-1} d x$ is a $\Gamma$-function,

$$
\int_{a}^{b}(x-\xi)^{k} e^{n \varphi(x)} d x= \begin{cases}\frac{2}{m}\left(\frac{-m !}{n \psi^{(m)}(\xi)}\right)^{\frac{1+k}{m}} e^{n \varphi(\xi)} \Gamma\left(\frac{1+k}{m}\right)\left(1+O\left(\frac{1}{n}\right)\right), \\ O\left(\left(\frac{1}{n}\right)^{\frac{1+k+m}{m}}\right), & \text { when } k \text { is even }\end{cases}
$$

\section{Regular relaxation oscillations in system (1.1)}

In this section, let $a \in\left(a_{1}, a_{0}\right)$ be such that the equilibrium point $\left(x_{e}, y_{e}\right)$ of system (1.1) lies on the middle branch of the critical curve. We will show that only regular relaxation oscillations can occur in this case.

Let $\frac{t-t_{0}}{\epsilon}=\tau$, with which, system (1.1) becomes

$$
\left\{\begin{array}{l}
\frac{d x}{d \tau}=g(y)-f(x), \\
\frac{d y}{d \tau}=\epsilon h(x, y, a) .
\end{array}\right.
$$


It then follows from Lemma 1 that

$$
\left\{\begin{array}{l}
y(\tau)-y_{0}=O(\epsilon), \\
x(\tau)-\bar{x}(\tau)=O(\epsilon),
\end{array} \quad 0 \leq \tau \leq T,\right.
$$

in which, $\bar{x}(\tau)$ is the solution to the following system,

$$
\frac{d \bar{x}}{d \tau}=g\left(y_{0}\right)-f(\bar{x}), \bar{x}(0)=x_{0},
$$

where $\left(x_{0}, y_{0}\right)$ is an initial value chosen satisfying $f\left(x_{0}\right) \neq g\left(y_{0}\right)$, inside the basin of attraction of $C O$, and outside the so-called singular periodic orbit $O A B C$ (see Figure 2).

The solution $\bar{x}(\epsilon)$ exists for all $\tau$. Hence, we can take $T$ arbitrarily large in (3.1). It thus follows from Lemma 2 that

$$
\left\{\begin{array}{l}
y(\tau)-y_{0}=o(1) \\
x(\tau)-\bar{x}(\tau)=o(1),
\end{array}\right.
$$

where $\tau^{*}(\epsilon)=\frac{\tau}{\delta(\epsilon)}, \delta(\epsilon)=o(1)$. Let $\bar{x}_{0}$ be chosen satisfies $g\left(y_{0}\right)-f\left(\bar{x}_{0}\right)=0$, then we have

$$
\bar{x}(\tau)=\bar{x}_{0}+O\left(\exp \left(-f^{\prime}\left(\bar{x}_{0}\right) \tau\right)\right) .
$$

Hence

$$
\bar{x}\left(\tau^{*}(\epsilon)\right)=\bar{x}_{0}+o(1) .
$$

This says that, after starting from $\left(x_{0}, y_{0}\right)$, the trajectory of system (1.1) evolves near the following orbit

$$
x=\bar{x}(\tau), \quad y=y_{0},
$$

and then penetrates into a small neighborhood of the curve $g(y)=f(x)$.

To see the dynamics near the critical curve, let

$$
g(y)=f(x)+\epsilon \phi_{0}(x, y)+\epsilon^{2} \phi_{1}(x, y)+\cdots,
$$

and then differentiating this formula with respect to $t$ yields

$$
g^{\prime}(y) \frac{d y}{d t}=f^{\prime}(x) \frac{d x}{d t}+\epsilon \frac{d \phi_{0}}{d t}+\cdots
$$

After substituting system (1.1) into this equality, to leading-order one gets

$$
\left\{\begin{array}{l}
\epsilon \frac{d x}{d t}=\epsilon \phi_{0}, \\
g^{\prime}(y) h(x, y, a)=f^{\prime}(x) \phi_{0},
\end{array}\right.
$$

i.e.,

$$
\phi_{0}=\frac{g^{\prime}(y) h(x, y, a)}{f^{\prime}(x)}
$$

Thus

$$
g(y)=f(x)+\epsilon\left[\phi_{0}+o(1)\right]
$$


One can see from (3.2) that, the slow flow evolves well (which means that the flow of system (1.1) evolves also well) near the critical curve until it enters into a small neighborhood of the turning point $x_{2}=0$ where $f^{\prime}(0)=0$. Since $g^{\prime}(0)>0$ and $h(0,0, a) \neq 0$ when $a \in\left(a_{1}, a_{0}\right)$, so the slow flow $\phi_{0}$ becomes unbounded in this small neighborhood. The ill-posedness of the slow flow results that the flow of system (1.1) may blow up. In this sense, we say that

$$
h(0,0, a) \neq 0, \quad a \in\left(a_{1}, a_{0}\right)
$$

is a necessary condition for the occurrence of blow-up of the flow of system (1.1).

To see whether the flow of system (1.1) really blows up when it enters the small neighborhood around the turning point $x=0$, let us write

$$
f^{\prime}(x)=x^{m-1} p(x), \quad p(x)>0,
$$

which yields

$$
f(x)=x^{m}\left(\frac{1}{m} p(0)+O(x)\right)
$$

and then introduce the following blow-up transformations,

$$
x=\epsilon^{\frac{1}{2 m-1}} \frac{\xi}{\delta^{*}(\epsilon)}, \quad y=\epsilon^{\frac{m}{2 m-1}} \eta, \quad t=\epsilon^{\frac{m}{2 m-1}} \tau+t_{0},
$$

where $\delta^{*}(\epsilon)=o(1)$ will be determined later, and $t_{0}$ denotes the moment when the flow enters into an $O(\epsilon)$-neighborhood of the turning point, after substituting (3.4) into equation (1.1), one gets

$$
\left\{\begin{aligned}
\frac{d \xi}{d \tau}= & g^{\prime}(0) \eta \delta^{*}+O\left(\epsilon^{\frac{m}{2 m-1}} \delta^{*} \eta^{2}\right)-\frac{1}{\delta^{*}(m-1)} \xi^{m}\left\{\frac{1}{m} p(0)+O\left(\frac{\epsilon^{\frac{1}{2 m-1}}}{\delta^{*}} \xi\right)\right\} \\
\frac{d \eta}{d \tau}= & h(0,0, a)+\epsilon^{\frac{1}{2 m-1}} \frac{\partial h}{\partial x}(0,0, a) \frac{\xi}{\delta^{*}}+\epsilon^{\frac{m}{2 m-1}} \frac{\partial h}{\partial y}(0,0, a) \eta \\
& +O\left(\epsilon^{\frac{2}{2 m-1}} \frac{\zeta^{2}}{\delta^{* 2}}+\epsilon^{\frac{2 m}{2 m-1}} \eta^{2}\right) .
\end{aligned}\right.
$$
get

Let $\delta^{*}(\epsilon)=O\left(\epsilon^{\frac{1}{m(2 m-1)}}\right)$ be negative and $\tau^{*}=\frac{\tau}{\left(\delta^{*}(\epsilon)\right)^{(m-1)}}$, with which, we

$$
\left\{\begin{aligned}
\frac{d \xi}{d \tau^{*}} & =g^{\prime}(0) \eta \delta^{* m}+O\left(\epsilon^{\frac{m}{2 m-1}} \delta^{* m} \eta^{2}\right)-\xi^{m}\left\{\frac{1}{m} p(0)+O\left(\frac{\epsilon^{\frac{1}{2 m-1}}}{\delta^{*}} \xi\right)\right\}, \\
\frac{d \eta}{d \tau^{*}} & =h(0,0, a) \delta^{*(m-1)}+\delta^{*(m-2)} \epsilon^{\frac{1}{2 m-1}} \frac{\partial h}{\partial x}(0,0, a) \xi \\
& +\delta^{*(m-1)} \cdot \epsilon^{\frac{m}{2 m-1}} \frac{\partial h}{\partial y}(0,0, a) \eta+\delta^{*(m-1)} O\left(\epsilon^{\frac{2}{2 m-1}} \frac{\zeta^{2}}{\delta^{* 2}}+\epsilon^{\frac{2 m}{2 m-1}} \eta^{2}\right),
\end{aligned}\right.
$$

which can be approximated by

$$
\left\{\begin{aligned}
\frac{d \xi}{d \tau^{*}} & =-\frac{1}{m} p(0) \xi^{m} \\
\frac{d \eta}{d \tau^{*}} & =0
\end{aligned}\right.
$$


with an $O\left(\epsilon^{\frac{m-1}{m(2 m-1)}}\right)$ accuracy. Equation (3.5) is solvable whose solutions are given by

$$
\hat{\xi}\left(\tau^{*}\right)=\frac{\xi^{0}}{\left(\frac{m-1}{m} p(0) \xi^{0(m-1)} \tau^{*}+1\right)^{\frac{1}{m-1}}}, \quad \hat{\eta}\left(\tau^{*}\right)=\eta^{0}
$$

where $\left(\xi^{0}, \eta^{0}\right)$ is an initial value.

Clearly, when $\tau^{*}$ reaches $\tau_{1}^{*}=-\left(\frac{m-1}{m} p(0) \xi^{0(m-1)}\right)^{-1}<0, \hat{\xi}\left(\tau^{*}\right)$ explodes, i.e., the flow of system (1.1) blows up there. Since then the flow leaves the neighborhood of the turning point $(0,0)$ along the fast fiber $y=0$ with an $O\left(\epsilon^{\frac{m}{2 m-1}}\right)$ distance.

Summarizing, after starting from the initial point $\left(x_{0}, y_{0}\right)$ with $g\left(y_{0}\right)-$ $f\left(x_{0}\right) \neq o(1)$ and inside the basin of attraction of $C O$, the flow of system (1.1) quickly enters into an $O(\epsilon)$-neighborhood of the stable branch $C O$, and then stays near $C O$ until it reaches near a small neighborhood of the turning point $O$ where the flow blows up and leaves along the fast fiber $y=0$ with an $O\left(\epsilon^{\frac{m}{2 m-1}}\right)$ distance. Afterward, the flow approaches to $A B$ and stays near $A B$ until it reaches near the turning point $B$, where it blows up again and jumps to $C O$ along the fast fiber $B C$ with an $O\left(\epsilon^{\frac{2}{3}}\right)$ distance. That is to say, the flow of system (1.1) spiraling around the singular periodic orbit $O A B C$ asymptotically, see Figure 2.

On the other hand, it can be proved that the equilibrium point $\left(x_{e}, y_{e}\right)$ is an unstable node by following Shen [21]. The flow of system (1.1) starting from near $\left(x_{e}, y_{e}\right)$ will spiral out and approaches to the singular periodic orbit $O A B C$ gradually. Till now, the existence of at least one relaxation oscillation near the singular periodic orbit $O A B C$ follows directly from the Poincare-Bendixson Theorem [6].

Theorem 1. If (A1) to (A4) and (3.3) hold, then when $a \in\left(a_{1}, a_{0}\right)$ and $\epsilon$ is sufficiently small, system (1.1) admits at least one relaxation oscillation near the singular periodic orbit $O A B C$. More precisely, the relaxation oscillations are near the slow branches $C O$ and $A B$ with an $O(\epsilon)$ distance, and near the fast fibers $B C$ and $O A$ with an $O\left(\epsilon^{\frac{2}{3}}\right)$ and an $O\left(\epsilon^{\frac{m}{2 m-1}}\right)$ distances respectively.

Remark 4. Theorem 1 shows that, under (A1) to (A4) and (3.3), when $a \in$ $\left(a_{1}, a_{0}\right)$, the flow of system (1.1) leaves the turning point $O$ near the fast fiber $y=0$ with an $O\left(\epsilon^{\frac{m}{2 m-1}}\right)$ distance. This result can be seen as a partial generalization of Theorem 2.1 in [14]. For the following general singular perturbation system,

$$
\left\{\begin{array}{l}
x^{\prime}=f(x, y, \mu, \epsilon), \\
y^{\prime}=\epsilon g(x, y, \mu, \epsilon),
\end{array}\right.
$$

Krupa and Szmolyan [14] proved via blow-up technique that, when the turning point $(0,0)$ is generic, the flow of this system will leave this turning point near the fast fiber $y=0$ with an $O\left(\epsilon^{\frac{2}{3}}\right)$ distance. 


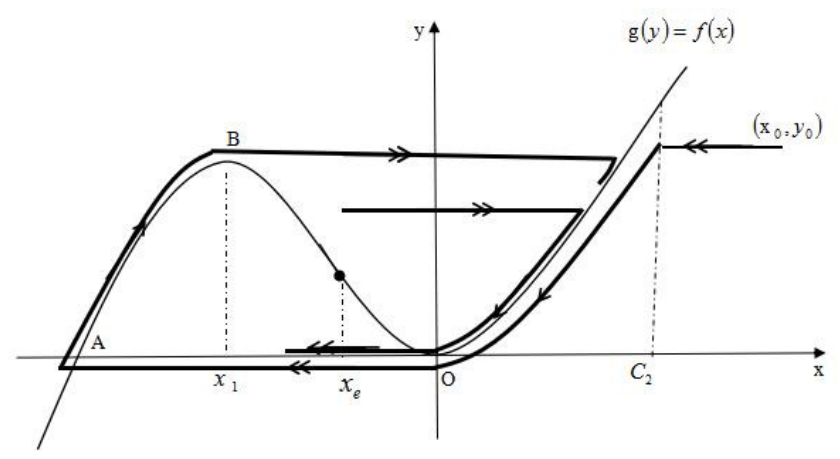

Figure 2. Schematic plots of two representative trajectories starting respectively inside and outside the singular periodic orbit OABC. In the figure, the large dot stands for the equilibrium.

\section{Canard relaxation oscillations in system (1)}

This section will consider the case $a \rightarrow a_{0}$, under which, the equilibrium point coincides with a critical point of the critical curve.

Let $g(y)-f(x)=\epsilon \phi$, then one gets from system (1.1) that

$$
\left\{\begin{array}{l}
\frac{d x}{d t}=\phi \\
\frac{d \phi}{d t}=\frac{1}{\epsilon}\left[-f^{\prime}(x) \phi+g^{\prime}(y) h(x, y, a)\right]
\end{array}\right.
$$

where $f^{\prime}(x)=x^{m-1} p(x)$ with $p(x)>0$. Let $\phi=-1 / p(x)+\epsilon \phi_{1}$, then system (4.1) becomes

$$
\left\{\begin{array}{l}
\frac{d x}{d t}=-\frac{1}{p(x)}+\epsilon \phi_{1} \\
\frac{d \phi_{1}}{d t}=\frac{x^{m-1}+g^{\prime}(y) h(x, y, a)}{\epsilon^{2}}+\frac{1}{\epsilon}\left(\frac{p^{\prime}(x)}{p^{3}(x)}-x^{m-1} p(x) \phi_{1}\right)-\frac{p^{\prime}(x)}{p^{2}(x)} \phi_{1},
\end{array}\right.
$$

i.e.

$$
\begin{aligned}
\frac{d \phi_{1}}{d x}= & \left(\frac{x^{m-1} p^{2}(x)}{\epsilon}+\frac{p^{\prime}(x)}{p(x)}+\epsilon p(x) \frac{d \phi_{1}}{d x}\right) \phi_{1} \\
& -\frac{x^{m-1} p(x)+g^{\prime}(y) h(x, y, a) p(x)}{\epsilon^{2}}-\frac{p^{\prime}(x)}{\epsilon p^{2}(x)} .
\end{aligned}
$$

Formally, the solution to equation (4.2) are given by

$$
\begin{aligned}
\phi_{1}= & e^{\int_{x_{0}}^{x} \frac{\xi^{m-1} p^{2}(\xi)}{\epsilon}+\frac{p^{\prime}(\xi)}{p(\xi)}+\epsilon p(\xi) \frac{d \phi_{1}}{d \xi} d \xi}\left(\int_{x_{0}}^{x}-\left(\frac{g^{\prime}(y) h(\xi, y, a) p(\xi)+\xi^{m-1} p(\xi)}{\epsilon^{2}}\right.\right. \\
& \left.\left.+\frac{p^{\prime}(\xi)}{\epsilon p^{2}(\xi)}\right) e^{-\int_{x_{0}}^{x} \frac{s^{m-1} p^{2}(s)}{\epsilon}+\frac{p^{\prime}(s)}{p(s)}+\epsilon p(s) \frac{d \phi_{1}}{d s} d s} d \xi+\phi_{1}\left(x_{0}\right)\right) .
\end{aligned}
$$


Denote

$$
\frac{d Q}{d x}=x^{m-1} p^{2}+\epsilon^{2} p \frac{\phi_{1}}{d x}
$$

then

$$
Q(x)=\hat{Q}(x)+\epsilon^{2} p \phi_{1}-\epsilon^{2} \int_{0}^{x} p^{\prime} \phi_{1} d \xi
$$

where $p^{\prime}=\frac{d p}{d \xi}$ and

$$
\hat{Q}(x)=\int_{0}^{x} \xi^{m-1} p^{2} d \xi
$$

Accordingly we can write

$$
\phi_{1}=\Phi_{1}\left(x, \phi_{1}\right)+\Phi_{2}\left(x, \phi_{1}\right)
$$

where

$$
\Phi_{1}\left(x, \phi_{1}\right)=\frac{p(x) \phi_{1}\left(x_{0}\right)}{p\left(x_{0}\right)} e^{\frac{1}{\epsilon} Q(x)-\frac{1}{\epsilon} Q\left(x_{0}\right)}
$$

and

$$
\Phi_{2}\left(x, \phi_{1}\right)=p(x) e^{\frac{1}{\epsilon} Q(x)} \int_{x_{0}}^{x}-\left(\frac{g^{\prime}(y) h(\xi, y, a)+\xi^{m-1}}{\epsilon^{2}}+\frac{p^{\prime}(\xi)}{\epsilon p^{3}(\xi)}\right) e^{-\frac{1}{\epsilon} Q(\xi)} d \xi .
$$

Let $x_{1}<0, x_{0}>0, x \in\left[x_{1}, x_{0}\right]$ and $U$ be a set of continuous functions $u(x)$ on $\left[x_{1}, x_{0}\right]$. Then we define an operator $L$ on $U$ by

$$
L u=\Phi_{1}(x, u)+\Phi_{2}(x, u), x \in\left[x_{1}, x_{0}\right] .
$$

When $u \in U$, since $Q(x)=\hat{Q}(x)+O\left(\epsilon^{2}\right)$, then

$$
\begin{aligned}
\Phi_{1}(x, \mu)= & \frac{p(x) \mu\left(x_{0}\right)}{p\left(x_{0}\right)} e^{\frac{1}{\epsilon}\left(\hat{Q}(x)-\hat{Q}\left(x_{0}\right)+O(\epsilon)\right)} \\
= & \frac{p(x) \mu\left(x_{0}\right)}{p\left(x_{0}\right)} e^{\frac{1}{\epsilon}\left(\hat{Q}(x)-\hat{Q}\left(x_{0}\right)\right)}(1+O(\epsilon)), \\
\Phi_{2}\left(x, \phi_{1}\right)= & -p(x) e^{\frac{1}{\epsilon} \hat{Q}(x)} \int_{x_{0}}^{x}\left(\frac{g^{\prime}(y) h(\xi, y, a)+\xi^{m-1}}{\epsilon^{2}}\right. \\
& \left.+\frac{p^{\prime}(\xi)}{\epsilon p^{3}(\xi)}\right) e^{-\frac{1}{\epsilon} \hat{Q}(\xi)} d \xi(1+O(\epsilon)) .
\end{aligned}
$$

Accordingly we can obtain

$$
L u=\hat{\phi_{1}}\{1+O(\epsilon)\}
$$

in which,

$$
\begin{aligned}
\hat{\phi_{1}}= & \Phi_{1}(x, 0)+\Phi_{2}(x, 0)=\frac{p(x) \phi_{1}\left(x_{0}\right)}{p\left(x_{0}\right)} e^{\left(\hat{Q}(x)-\hat{Q}\left(x_{0}\right)\right) / \varepsilon} \\
& +\frac{p(x)}{\epsilon} e^{\frac{1}{\epsilon} \hat{Q}(x)} \int_{x}^{x_{0}}\left(\frac{g^{\prime}(\hat{y}) h(\xi, \hat{y}, a)+\xi^{m-1}}{\epsilon}+\frac{p^{\prime}(\xi)}{p^{3}(\xi)}\right) e^{-\frac{1}{\epsilon} \hat{Q}(\xi)} d \xi,
\end{aligned}
$$


where $\hat{y}=\hat{y}(x)$ is determined by

$$
g(\hat{y})=f(x)-\epsilon / p(x) .
$$

So far we know that if $\hat{\phi}_{1}(x)$ is bounded on $\left[x_{1}, x_{0}\right]$, then according to the contraction mapping principle, the operator $L$ admits a unique fixed point, which corresponds to the solution to equation (4.2) with

$$
\phi_{1}(x)=\hat{\phi}_{1}\{1+o(1)\} .
$$

Hence, if $\hat{\phi}_{1}(x)$ is bounded, then equation (4.2) has bounded solutions on $\left[x_{1}, x_{0}\right]$, which states that canard solutions in system (1.1) exist. In the following, we show that, under certain conditions, $\hat{\phi}_{1}(x)$ is bounded.

Since $h\left(0,0, a_{0}\right)=0$, so by Taylor expansion and Lemma 3, it can be deduced that

$$
\begin{aligned}
& \int_{x}^{x_{0}}\left(\frac{g^{\prime}(\hat{y}) h(\xi, \hat{y}, a)+\xi^{m-1}}{\epsilon}+\frac{p^{\prime}(\xi)}{p^{3}(\xi)}\right) e^{-\frac{1}{\epsilon} \hat{Q}(\xi)} d \xi \\
& =\int_{x}^{x_{0}} e^{-\frac{1}{\epsilon} \int_{0}^{\xi} s^{m-1} p^{2}(s) d s}\left[\frac{p^{\prime}(\xi)}{p^{3}(\xi)}+\sum_{i=1}^{l} g^{\prime}(\hat{y}) \frac{1}{i !} \frac{\partial^{i} h}{\partial \xi^{i}} / \epsilon\right. \\
& +\frac{\frac{\partial h}{\partial a}\left(a-a_{0}\right) g^{\prime}(\hat{y})+o\left(\xi^{l},\left|a-a_{0}\right|\right)+\xi^{m-1}}{\epsilon} d \xi \\
& =\frac{1}{\epsilon}\left\{\sum_{i=1}^{l / 2} g^{\prime}(\hat{y}) \frac{1}{(2 i) !} \frac{\partial^{2 i} h}{\partial x^{2 i}} \frac{2}{m}\left(\frac{\epsilon m}{p^{2}(0)}\right)^{\frac{2 i+1}{m}} \Gamma\left(\frac{2 i+1}{m}\right)+O\left(\epsilon^{\frac{m+1}{m}}\right)\right. \\
& +\frac{\partial h}{\partial a}\left(a-a_{0}\right) g^{\prime}\left(g^{-1}\left(\frac{-\epsilon}{p(0)}\right)\right) \frac{2}{m}\left(\frac{\epsilon m}{p^{2}(0)}\right)^{\frac{1}{m}} \Gamma\left(\frac{1}{m}\right)(1+O(\epsilon)) \\
& \left.+\frac{p^{\prime}(0)}{p^{3}(0)} \frac{2 \epsilon}{m}\left(\frac{\epsilon m}{p^{2}(0)}\right)^{\frac{1}{m}} \Gamma\left(\frac{1}{m}\right)(1+O(\epsilon))\right\} \\
& =\frac{2}{\epsilon m}\left(\frac{\epsilon m}{p^{2}(0)}\right)^{\frac{1}{m}}\left[\sum_{i=1}^{\frac{m}{2}} g^{\prime}(\hat{y}) \frac{1}{(2 i) !} \frac{\partial^{2 i} h}{\partial x^{2 i}}\left(\frac{\epsilon m}{p^{2}(0)}\right)^{\frac{2 i}{m}} \Gamma\left(\frac{2 i+1}{m}\right)\right. \\
& \left.+\frac{\partial h}{\partial a}\left(a-a_{0}\right) g^{\prime}\left(g^{-1}\left(\frac{-\epsilon}{p(0)}\right)\right) \Gamma\left(\frac{1}{m}\right)+\frac{\epsilon p^{\prime}(0)}{p^{3}(0)} \Gamma\left(\frac{1}{m}\right)+O(\epsilon)\right],
\end{aligned}
$$

where $l$ is an even number, $g^{-1}$ is the inverse function of $g$, all the partial derivatives take their values at $\left(0, \hat{y}, a_{0}\right), o\left(\xi^{l},\left|a-a_{0}\right|\right)$ denotes the Lagrange remainder term, and $\Gamma$ is the Gamma function. Thus,

$$
\begin{aligned}
\hat{\phi_{1}}(x)= & \frac{p(x) \phi_{1}\left(x_{0}\right)}{p\left(x_{0}\right)} e^{\frac{1}{\epsilon} \hat{Q}(x)-\frac{1}{\epsilon} \hat{Q}\left(x_{0}\right)}+\frac{p(x)}{\epsilon} e^{\frac{1}{\epsilon} \hat{Q}(x)} \frac{2}{\epsilon m}\left(\frac{\epsilon m}{p^{2}(0)}\right)^{\frac{1}{m}} \\
& \times\left[\sum_{i=1}^{m / 2} g^{\prime}(\hat{y}) \frac{1}{(2 i) !} \frac{\partial^{2 i} h}{\partial x^{2 i}}\left(\frac{\epsilon m}{p^{2}(0)}\right)^{\frac{2 i}{m}} \Gamma\left(\frac{2 i+1}{m}\right)\right. \\
& \left.+\frac{\partial h}{\partial a}\left(a-a_{0}\right) \times g^{\prime}\left(g^{-1}\left(\frac{-\epsilon}{p(0)}\right)\right) \Gamma\left(\frac{1}{m}\right)+\frac{\epsilon p^{\prime}(0)}{p^{3}(0)} \Gamma\left(\frac{1}{m}\right)+O(\epsilon)\right] .
\end{aligned}
$$


Denote

$$
\begin{aligned}
G(a, \epsilon)= & \sum_{i=1}^{\frac{m}{2}} g^{\prime}(\hat{y}) \frac{1}{(2 i) !} \frac{\partial^{2 i} h}{\partial x^{2 i}}\left(\frac{\epsilon m}{p^{2}(0)}\right)^{\frac{2 i}{m}} \Gamma\left(\frac{2 i+1}{m}\right) \\
& +\frac{\partial h}{\partial a}\left(a-a_{0}\right) g^{\prime}\left(g^{-1}\left(\frac{-\epsilon}{p(0)}\right)\right) \Gamma\left(\frac{1}{m}\right)+\frac{\epsilon p^{\prime}(0)}{p^{3}(0)} \Gamma\left(\frac{1}{m}\right)+O(\epsilon),
\end{aligned}
$$

thus,

$$
\hat{\phi}(x)=\frac{p(x) \phi_{1}\left(x_{0}\right)}{p\left(x_{0}\right)} e^{\frac{1}{\epsilon} \hat{Q}(x)-\frac{1}{\epsilon} \hat{Q}\left(x_{0}\right)}+\frac{p(x)}{\epsilon} e^{\frac{1}{\epsilon} \hat{Q}(x)} \frac{2}{\epsilon m}\left(\frac{\epsilon m}{p^{2}(0)}\right)^{\frac{1}{m}} G(a, \epsilon) .
$$

Since $\frac{\partial h}{\partial a}\left(0,0, a_{0}\right) \neq 0$, it thus can be verified that $G\left(a_{0}, 0\right)=0$ and $\frac{\partial G}{\partial a}\left(a_{0}, 0\right) \neq$ 0 . By implicit function theorem, there exists a unique function $a_{c}=a_{c}(\epsilon)$ satisfying $a_{c}(0)=a_{0}$ such that $G\left(a_{c}(\epsilon), \epsilon\right)=0$.

Let us take the breaking parameter $a$ as

$$
a=a_{c}(\epsilon)+\sigma \epsilon^{2-\frac{1}{m}} e^{-\frac{k^{2}}{\epsilon}}
$$

where $\sigma$ and $k$ are two constants, it then follows from equation (4.3) that

$$
\begin{aligned}
\hat{\phi}(x)= & \frac{p(x) \phi_{1}\left(x_{0}\right)}{p\left(x_{0}\right)} e^{\frac{1}{\epsilon} \hat{Q}(x)-\frac{1}{\epsilon} \hat{Q}\left(x_{0}\right)}+\sigma \frac{\partial h}{\partial a} g^{\prime}\left(g^{-1}\left(\frac{-\epsilon}{p(0)}\right)\right) \\
& \times \Gamma\left(\frac{1}{m}\right) \frac{2 p(x)}{m}\left(\frac{\epsilon m}{p^{2}(0)}\right)^{\frac{1}{m}} e^{\frac{1}{\epsilon}\left(\hat{Q}(x)-k^{2}\right)} .
\end{aligned}
$$

Let $\left(x_{0}, y_{0}\right)$ be an initial point near the right branch of the critical curve, $x_{0}^{*}<0$ be a root of the equation $\hat{Q}\left(x_{0}\right)=\hat{Q}\left(x_{0}^{*}\right)$, and $k$ be an arbitrary constant such that $x_{k}>0$ and $x_{k}^{*} \in(A, 0)$ are respectively the roots solved from the equations $\hat{Q}\left(x_{k}\right)=\hat{Q}\left(x_{k}^{*}\right)=k^{2}$, then we can obtain from (4.4) that $\phi_{1}(x)$ is bounded provided $\max \left\{x_{k}^{*}, x_{0}^{*}\right\} \leq x \leq \min \left\{x_{0}, x_{k}\right\}$. Accordingly, system (1.1) has a canard solution satisfying

$$
g(y)=f(x)-\frac{\epsilon}{p(x)}+\epsilon^{2} \phi_{1}(x) .
$$

This canard solution will form a canard relaxation oscillation finally.

Moreover, it can be seen from (4.4) that the first term is dominant if $x_{0}<$ $x_{k}$, and otherwise the second one will be dominant if $x_{0}>x_{k}$.

For the former case, after starting from the basin of attraction of the right branch of the critical curve, all the trajectories of system (1.1) enters into an $O(\epsilon)$-neighborhood of the critical curve $g(y)=f(x)$, after going through the turning point, they stay near the repelling branch for a constant distance until it jumps off at $x=x_{0}^{*}$. The trajectories jump to the right if $\phi_{1}\left(x_{0}\right)>0$ and to the left if $\phi_{1}\left(x_{0}\right)<0$.

For the latter case, after starting from the basin of attraction of the right branch of the critical curve, all trajectories enter into an $O(\epsilon)$-neighborhood of $g(y)=f(x)$ at $x=x_{0}>x_{k}$ and then stay near the attracting and repelling 
branches of the critical curve until they jump off at $x=x_{k}^{*}<0$. The trajectories jump off to the right and the left respectively when $\sigma \frac{\partial h}{\partial a}(0,0,0)>0$ and $\sigma \frac{\partial h}{\partial a}(0,0,0)<0$, which respectively yields the birth of canard relaxation oscillations with or without head, see Figure 3.

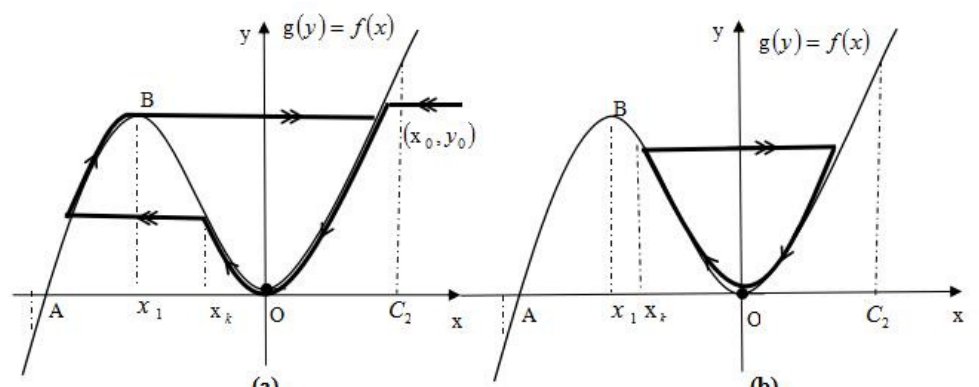

(a)

(b)

Figure 3. Schematic plots of canard relaxation oscillations with or without head. (a): $\sigma \frac{\partial h}{\partial a}(0,0,0)<0 ;(\mathrm{b}): \sigma \frac{\partial h}{\partial a}(0,0,0)>0$.

Summing up, we have the following conclusion.

Theorem 2. If (A1) to (A5) hold, when $\epsilon$ is sufficiently small and $a=$ $a_{c}(\epsilon)+\sigma \epsilon^{2-\frac{1}{m}} e^{-\frac{k^{2}}{\epsilon}}$ with $a_{c}(0)=a_{0}$, where $\sigma$ and $k$ are two constants, then system (1.1) has at least one canard relaxation oscillation with or without head depending on the breaking parameter and the initial values (see Figure 3).

\section{$5 \quad$ Examples}

\subsection{A FitzHugh-Nagumo equation}

Consider a FitzHugh-Nagumo (FHN) equation as follows,

$$
\left\{\begin{array}{l}
\epsilon \dot{u}=v-u^{3} / 3+u+p \\
\dot{v}=-(v+r u-a) / s
\end{array}\right.
$$

where $0<\epsilon \ll 1, p, s, r$ and $a$ are the parameters of the model. System (5.1) is a prototypical FitzHugh-Nagumo equation describing the biological phenomena with neuronal excitability and spike generating mechanism.

By the translation as follows, $u=x+1, v=y$, the extreme point $(-1,0)$ of the critical point is then transferred to the origin, and accordingly we have

$$
\left\{\begin{array}{l}
\epsilon \dot{x}=y-\left((x+1)^{3} / 3-x-1-p\right), \\
\dot{y}=-(y+r(x+1)-a) / s
\end{array}\right.
$$

Therefore, in this example we have

$$
g(y)=y, \quad f(x)=(x+1)^{3} / 3-x-1-p
$$


and

$$
h(x, y, a)=-\frac{1}{s}(y+r(x+1)-a)
$$

For system (5.2), it can be observed that:

1) When $p=-\frac{2}{3}$, the critical curve $y=f(x)$ passes through the origin, and there are two generic extreme points $x=-2$ and $x=0$ with $f^{\prime}(-2)=0$, $f^{\prime \prime}(-2)<0, f^{\prime}(0)=0$ and $f^{\prime \prime}(0)>0$;

2) Let $s>0, r<-3$ be fixed. If $a<r$, then inside the singular periodic orbit formed by two slow orbits and two fast fibers (bounded by four points: $(0,0),(-3,0),\left(-2, \frac{4}{3}\right)$ and $\left.\left(1, \frac{4}{3}\right)\right)$, system $(5.2)$ has a unique equilibrium $\left(x_{e}, y_{e}\right)$ on the middle branch of the critical curve. In this case, the turning point $(0,0)$ is a jump point.

3) Let $s>0, r<-3$ be fixed. If $a=r$, then system (5.2) has a unique equilibrium $\left(x_{e}, y_{e}\right)$ coinciding with the extreme point $(0,0)$. In this case, the turning point $(0,0)$ is a canard point.

4) $h(0,0, r, a)=0, \quad \frac{\partial h(0,0, r, a)}{\partial a} \neq 0$.

By following Theorems 1-2, we have

Theorem 3. Let $p=-\frac{2}{3}, s>0$ and $r<-3$ be fixed, and $\epsilon$ is sufficiently small, we then have the following conclusions:

1) If $a<r$, then system (5.2) has at least one regular relaxation oscillation near the singular periodic orbit described above;

2) If $a=a_{c}(\epsilon)+\sigma \epsilon^{\frac{3}{2}} e^{-\frac{k^{2}}{\epsilon}}$ where $a_{c}(0)=r$, and $\sigma, k$ are two constants, then system (5.2) has at least one canard relaxation oscillation with or without head depending on the sign of the quantity as follows,

$$
\sigma \frac{\partial h}{\partial a}(0,0, r)=\frac{\sigma}{s}
$$

where $a_{c}(\epsilon)$ is determined by the following equation

$$
\begin{aligned}
G(a, \epsilon)= & \left.\frac{a-r}{2 \sqrt{2} s}+\frac{\epsilon}{4 \sqrt{2} s}+\frac{\epsilon}{16 \sqrt{2} s}\right) \Gamma\left(\frac{1}{2}\right) \\
& +\frac{\epsilon(s+r)}{\sqrt{2} s} \Gamma\left(\frac{3}{2}\right)+\frac{7 \epsilon^{2}}{8 \sqrt{2} s^{2}} \Gamma\left(\frac{5}{2}\right)+\frac{\epsilon^{3}}{32 \sqrt{2} s} \Gamma\left(\frac{7}{2}\right)+O\left(\epsilon^{2}\right)=0 .
\end{aligned}
$$

\subsection{A predator-prey model with Holling-II response}

Consider the following classical two-dimensional predator-prey model with Holling-II response,

$$
\left\{\begin{array}{l}
\dot{x}=x(1-x)-\frac{x y}{\lambda+x}, \\
\dot{y}=\epsilon y\left(-\mu+\frac{x}{\lambda+x}\right),
\end{array}\right.
$$

in which, $0<\lambda<1, \mu>0$ are two regular parameters, and $0<\epsilon \ll 1$ is the singular parameter. The existence of the regular and singular parameters depends on the specific biological settings. 
Let $\epsilon t=\tau$, one gets the associated slow system,

$$
\left\{\begin{array}{l}
\epsilon x^{\prime}=x(1-x)-\frac{x y}{\lambda+x}, \\
y^{\prime}=y\left(-\mu+\frac{x}{\lambda+x}\right) .
\end{array}\right.
$$

By introducing the following new time scale, $\tau=(\lambda+x) \tilde{t}$, by which, equation (5.4) can be changed to a polynomial system, namely

$$
\left\{\begin{array}{l}
\epsilon \frac{d x}{d \tilde{t}}=x(1-x)(\lambda+x)-x y \\
\frac{d y}{d \tilde{t}}=y(-\mu(\lambda+x)+x)
\end{array}\right.
$$

Let

$$
x=\frac{\mu \lambda}{1-\mu} \bar{x}, y=\mu \lambda \bar{y}, \tilde{t}=\frac{1}{\mu \lambda} \bar{t}
$$

then system (5.5) becomes

$$
\left\{\begin{array}{l}
\epsilon \frac{d \bar{x}}{d \bar{t}}=\frac{1}{\mu} \bar{x}+\frac{1-\lambda}{1-\mu} \bar{x}^{2}-\frac{\lambda \mu}{(1-\mu)^{2}} \bar{x}^{3}-\overline{x y} \\
\frac{d \bar{y}}{d \bar{t}}=\overline{x y}-\bar{y}
\end{array}\right.
$$

i.e.

$$
\left\{\begin{array}{l}
\epsilon \frac{d \bar{x}}{d \bar{t}}=a_{1} \bar{x}+a_{2} \bar{x}^{2}-a_{3} \bar{x}^{3}-\overline{x y} \\
\frac{d \bar{y}}{d \bar{t}}=\overline{x y}-\bar{y}
\end{array}\right.
$$

where

$$
a_{1}=\frac{1}{\mu}, a_{2}=\frac{1-\lambda}{1-\mu}, a_{3}=\frac{\lambda \mu}{(1-\mu)^{2}} .
$$

Let us further set $\bar{x}=e^{\overline{\bar{x}}}, \bar{y}=e^{\overline{\bar{y}}}$ and $\bar{t}=-\overline{\bar{t}}$, then we get

$$
\left\{\begin{array}{l}
\epsilon \frac{d x}{d t}=\left(e^{y}-a_{1}\right)-\left(a_{2} e^{x}-a_{3} e^{2 x}\right), \\
\frac{d y}{d t}=1-e^{x}
\end{array}\right.
$$

which has been written in a standard singular perturbation generalized Liénard systems, where we still use $x, y$ and $t$ to denote the dependent and independent variables for simplicity. Let

$$
\tilde{x}=x+\ln \frac{a_{2}}{2 a_{3}}, \quad \tilde{y}=y+\ln \left(a_{1}+\frac{a_{2}^{2}}{4 a_{3}}\right),
$$

by which, we obtain

$$
\left\{\begin{array}{l}
\epsilon \frac{d x}{d t}=\left(a_{1}+\frac{a_{2}^{2}}{4 a_{3}}\right) e^{y}-\left(a_{1}+\frac{a_{2}^{2}}{2 a_{3}} e^{x}-\frac{a_{2}^{2}}{4 a_{3}} e^{2 x}\right) \\
\frac{d y}{d t}=1-\frac{a_{2}}{2 a_{3}} e^{x}
\end{array}\right.
$$


where, for simplicity, the bars have been omitted.

In this example, we have

$$
g(y)=\left(a_{1}+\frac{a_{2}^{2}}{4 a_{3}}\right) e^{y}, \quad f(x)=a_{1}+\frac{a_{2}^{2}}{2 a_{3}} e^{x}-\frac{a_{2}^{2}}{4 a_{3}} e^{2 x}, \quad h(x)=1-\frac{a_{2}}{2 a_{3}} e^{x} .
$$

For system (5.6), one can see that

1) $x=0$ is a generic extreme point of the following critical curve

$$
\left(a_{1}+\frac{a_{2}^{2}}{4 a_{3}}\right) e^{y}=a_{1}+\frac{a_{2}^{2}}{2 a_{3}} e^{x}-\frac{a_{2}^{2}}{4 a_{3}} e^{2 x}
$$

with

$$
f^{\prime}(0)=0, \quad f^{\prime \prime}(0)=-\frac{a_{2}^{2}}{2 a_{3}}<0 .
$$

Therefore, near $x=0$, we can write $f^{\prime}(x)=x p(x)$, where $p(x)>0$.

2) $g(0)=f(0)=\frac{a_{2}^{2}}{4 a_{3}}$, and $g^{\prime}(y)=\left(a_{1}+\frac{a_{2}^{2}}{4 a_{3}}\right) e^{y}>0$.

3) System (5.6) has a singular point

$$
\left(x_{e}, y_{e}\right)=\left(\ln \frac{2 a_{3}}{a_{2}}, \ln \frac{4 a_{2} a_{3}-4 a_{3}^{2}+4 a_{1} a_{3}}{4 a_{1} a_{3}+a_{2}^{2}}\right) .
$$

4) As $a_{2} \rightarrow 2 a_{3},\left(x_{e}, y_{e}\right) \rightarrow(0,0)$, which is a canard point. When the flow of system (5.3) gets through a small neighborhood of the canard point, it displays the feature of delay loss of stability. By section 3, the position where the flow blows up is at $x=x_{k}$, which can be solved from the following equation

$$
\hat{Q}=\int_{0}^{x} \xi p^{2}(\xi) d \xi=k^{2} .
$$

If the flow extends to the right, it forms a canard relaxation oscillation without head, and if to the left, it will stay near $x=0$ and display the feature of delay loss of stability again when it passes through the transcritical bifurcation point, and finally forms another canard relaxation oscillation, see Figure 4.

5) Let $a=\frac{2 a_{3}}{a_{2}}=\frac{(1-\lambda)(1-\mu)}{2 \lambda \mu}$, then it can be verified that

$$
h(x, a)=1-a e^{x}, h(0,1)=1-a e^{x}=0, \frac{\partial h}{\partial a}(0,1)=-1 \neq 0 .
$$

From Section 3, we have the following conclusion:

Theorem 4. When $\epsilon$ is sufficiently small and

$$
a=\frac{(1-\lambda)(1-\mu)}{2 \lambda \mu}=a_{c}(\epsilon)+\sigma \epsilon^{\frac{3}{2}} e^{-\frac{k^{2}}{\epsilon}},
$$

where $a_{c}(0)=1$ and $\sigma, k$ are two constants, then system (5.3) has at least one canard solution with or without head, see Figure 4 , where $a_{c}(\epsilon)$ is determined by the following equation,

$G\left(a_{c}(\epsilon)\right)=\frac{\sqrt{2}\left(a_{c}(\epsilon)-1\right)}{p(0)} g^{\prime}\left(g^{-1}\left(\frac{-\epsilon}{p(0)}\right)\right) \Gamma\left(\frac{1}{2}\right)+O\left(\epsilon^{\frac{5}{2}}\right)+\sqrt{2} \epsilon \frac{p^{\prime}(0)}{p^{4}(0)} \Gamma\left(\frac{1}{2}\right)=0$. 


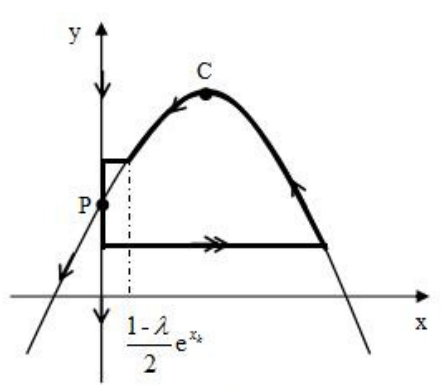

(a)

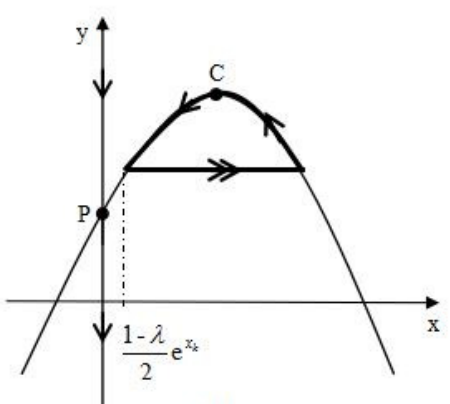

(b)

Figure 4. Schematic plots of canard relaxation oscillations in system (5.3). (a): $\sigma<0 ;(\mathrm{b}): \sigma>0$.

\section{Acknowledgements}

This work was supported by NSFC (No.11201072, 11401229), NSF of Fujian Province (No. 2015J01004, 2014J01002) and Nonlinear Analysis Innovation Team (IRTL 1206) funded by FJNU. The authors would also like to thank the referees for the proposed improvements.

\section{References}

[1] E. Benoit, J.L. Callot, F. Diener, M. Diener et al. Chasse au canard (première partie). Collectanea Mathematica, 32(1):37-76, 1981.

[2] X. Chen, P. Yu, M. Han and W. Zhang. Canard solutions of two-dimensional singularly perturbed systems. Chaos, Solitons \& Fractals, 23(3):915-927, 2005. https://doi.org/10.1016/S0960-0779(04)00339-X.

[3] P. De Maesschalck. Planar canards with transcritical intersections. Acta Applicandae Mathematicae, 137(1):159-184, 2015. https://doi.org/10.1007/s10440014-9994-9.

[4] P. De Maesschalck and F. Dumortier. Classical Liénard equations of degree $\mathrm{n} \geq 6$ can have $\left[\frac{n-1}{2}\right]+2$ limit cycles. Journal of Differential Equations, 250(4):21622176, 2011. https://doi.org/10.1016/j.jde.2010.12.003.

[5] F. Dumortier. Slow divergence integral and balanced canard solutions. Qualitative Theory of Dynamical Systems, 10(1):65-85, 2011. https://doi.org/10.1007/s12346-011-0038-9.

[6] F. Dumortier, J. Llibre and J.C. Artés. Qualitative theory of planar differential systems. Springer, 2006.

[7] F. Dumortier, D. Panazzolo and R. Roussarie. More limit cycles than expected in Liénard equations. Proceedings of the American Mathematical Society, 135(6):1895-1904, 2007. https://doi.org/10.1090/S0002-9939-07-08688-1.

[8] F. Dumortier and R. Roussarie. Bifurcation of relaxation oscillations in dimension two. Proceedings of the American Mathematical Society, 19(4):631-674, 2007. 
[9] F. Dumortier and R.H. Roussarie. Canard cycles and center manifolds, volume 577. American Mathematical Soc., 1996.

[10] W. Eckhaus. Relaxation oscillations including a standard chase on French ducks. In Asymptotic Analysis II, pp. 449-494. Springer, 1983. https://doi.org/10.1007/BFb0062381.

[11] J. Grasman. Relaxation oscillation. In Robert A. Meyers(Ed.), Mathematics of Complexity and Dynamical Systems, pp. 1475-1488. Springer, 2012. https://doi.org/10.1007/978-1-4614-1806-1_93.

[12] M. Han, Y. Tian and P. Yu. Small-amplitude limit cycles of polynomial Liénard systems. Science China Mathematics, 56(8):1543-1556, 2013. https://doi.org/10.1007/s11425-013-4618-9.

[13] S.-B. Hsu and J. Shi. Relaxation oscillator profile of limit cycle in predator-prey system. Disc. Cont. Dyna. Syst.-B, 11:893-911, 2009.

[14] M. Krupa and P. Szmolyan. Extending geometric singular perturbation theory to nonhyperbolic points - fold and canard points in two dimensions. SIAM Journal on Mathematical Analysis, 33(2):286-314, 2001. https://doi.org/10.1137/S0036141099360919.

[15] C. Li. Duck solutions: A new kind of bifurcation phenomenon in relaxation oscillations. Acta Mathematica Sinica, 12(1):89-104, 1996.

[16] C. Li. Duck solutions problem in singularly perturbed problems. Sci. China: Series A, 29(12):1084-1093, 1999.

[17] C. Li and H. Zhu. Canard cycles for predator-prey systems with Holling types of functional response. Journal of Differential Equations, 254(2):879-910, 2013. https://doi.org/10.1016/j.jde.2012.10.003.

[18] A. Lins, W. De Melo and C.C. Pugh. On Liénard's equation. In Geometry and topology, pp. 335-357. Springer, 1977. https://doi.org/10.1007/BFb0085364.

[19] W. Liu, D. Xiao and Y. Yi. Relaxation oscillations in a class of predatorprey systems. Journal of Differential Equations, 188(1):306-331, 2003. https://doi.org/10.1016/S0022-0396(02)00076-1.

[20] R. Roussarie. Putting a boundary to the space of Liénard equations. Discrete and Continuous Dynamical Systems, 17(2):441, 2007. https://doi.org/10.3934/dcds.2007.17.441.

[21] J. Shen. Canard limit cycles and global dynamics in a singularly perturbed predator-prey system with non-monotonic functional response. Nonlinear Analysis: Real World Applications, 31:146-165, 2016. https://doi.org/10.1016/j.nonrwa.2016.01.013.

[22] S Van Gils, M Krupa and P Szmolyan. Asymptotic expansions using blow-up. Zeitschrift für angewandte Mathematik und Physik ZAMP, 56(3):369-397, 2005.

[23] Y. Xiong and M. Han. New lower bounds for the Hilbert number of polynomial systems of Liénard type. Journal of Differential Equations, 257(7):2565-2590, 2014. https://doi.org/10.1016/j.jde.2014.05.058. 